\title{
Yeni Morfolinoetil-Sübstitüyeli (NHC)PdBr2(3-Kloropiridin) Kompleksleri: Sentez, Karakterizasyon ve Suzuki-Miyaura Reaksiyonundaki Katalitik Aktivite
}

\author{
Aydın AKTAŞ⿻ ${ }^{* 1}$ \\ 1İnönü Üniversitesi, Fen Edebiyat Fakültesi, Kimya Bölümü, 44280, Malatya, Türkiye
}

(Alınıș / Received: 30.10.2018, Kabul / Accepted: 05.04.2019, Online Yayınlanma / Published Online: 30.08.2019)

\section{Anahtar Kelimeler}

Fenilboronik asit, N-Heterosiklik karbenler, PEPPSI kompleksleri, Suzuki-Miyaura reaksiyonu
Özet: Son yıllarda, organometalik bileşiklerle çalışan kimyagerler, Pd-PEPPSI komplekslerine daha fazla ilgi duymaktadırlar. Bu çalışma; 2-morfolinoetil sübstitüyeli Pd-PEPPSI komplekslerinin sentezini ve bunların Suzuki-Miyaura çaprazeşleşme reaksiyonundaki katalitik aktivitelerini içerir. Sentezlenen Pd-PEPPSI kompleksleri; 2-morfolinoetil sübstitüyeli N-heterosiklik karben (NHC) öncüleri, paladyum klorür ve 3-kloropiridin'den hazırlanmıștır. Bu kompleksler, ${ }^{1} \mathrm{H}$ NMR, ${ }^{13} \mathrm{C}$ NMR, FTIR spektroskopisi ve elementel analiz teknikleri kullanılarak karakterize edilmiştir. Ayrıca, sentezlenen Pd-PEPPSI kompleksleri, Suzuki-Miyaura çaprazeşleşme reaksiyonunda katalizör olarak incelenmiş ve bu reaksiyonda iyi aktivite sergiledikleri gözlenmiştir.

\section{New Morpholinoethyl-Substituted (NHC)PdBr2(3-Chloropyridine) Complexes: Synthesis, Characterization and Catalytic Activity in the Suzuki-Miyaura Reaction}

\section{Keywords}

Phenylboronic acid, $\mathrm{N}$-Heterocyclic carbenes, PEPPSI complexes, Suzuki-Miyaura reaction

\begin{abstract}
Recently, chemists studying with organometallic compounds are increasingly interested in Pd-PEPPSI complexes. This study contains the synthesis of the 2-morpholinoethyl-substituted Pd-PEPPSI complexes and their catalytic activity in the Suzuki-Miyaura reaction. Synthesized the Pd-PEPPSI complexes have been prepared from the 2-morpholinoethyl-substituted N-heterocyclic carbene (NHC) precursors, palladium chloride and 3-chloropyridine. These complexes have been characterized by using ${ }^{1} \mathrm{H}$ NMR, ${ }^{13} \mathrm{C}$ NMR, FTIR spectroscopy and elementel analysis techniques. Also, synthesized the Pd-PEPPSI complexes have been examined as catalysts in the Suzuki-Miyaura cross-coupling reaction with arylboronic acid derivatives and have shown to exhibit well activity in this reaction.
\end{abstract}

\section{Giriş}

Son yllarda Pd-temelli komplekslerden Pd-PEPPSI kompleksleri, organometalik kimyacıların dikkatini çekmektedir. Pd-PEPPSI kompleksleri, iki halojen atomu, bir piridin türevi ve $\mathrm{Pd}$ merkez atomuna koordine olmuş bir NHC ligandı içeren kararlı organometalik bileşiklerdir. Bu bileşikler PEPPSI (Pyridine-Enhanced Precatalyst Preparation Stabilization and Initiation) olarak adlandırılmıştır [1]. Pd-PEPPSI kompleksleri, 2005 yılında Organ ve arkadaşları tarafından sentezlendikten sonra C-C bağ oluşum reaksiyonlarında aktif katalizörler olarak kullanılmıştır [2]. Organopaladyum bileşikleri olarak davranan bu bileşikler, Suzuki-Miyaura [3], Negishi [4], Mizoroki-Heck [3], Stille [5], Sonogashira [6], Hiyama [7], Kumada-Corriu [8], ve doğrudan arillasyon reaksiyonu $[9,10]$, gibi C-C bağ oluşum reaksiyonları ve aminasyon [11] gibi C-heteroatom bağ oluşum reaksiyonlarında aktif katalizörler olarak kullanılırlar.

Aslında Pd-PEPPSI komplekslerinin en önemli özelliği, $N$-heterosiklik karben (NHC) ligandlarını içermesidir. Bu ligandlar güçlü $\sigma$-verici ve zayıf $\pi$-alıcl özelliklerinin yanı sıra hava ve neme karșı kararlılıkları gibi önemli özelliklere sahiptir [8, 1213]. Bununla birlikte, bu ligandların elektronik ve yapısal özellikleri arzu edilen seviyelere ayarlanabilmektedir. [14, 15]. Pd-PEPPSI komplekslerinde yer alan NHC ligandları varlığının, katalitik döngüdeki oksidatif katılmayı (elektronik etki) ve indirgen eliminasyonu (yapısal özellik) kolaylaştırarak, Pd-PEPPSI komplekslerinin katalitik aktivitelerini arttırdığını söyleyebiliriz [16]. 
Suzuki-Miyaura çapraz-eşleşme reaksiyonu, $\mathrm{Pd}$ PEPPSI komplekslerinin katalizör olarak kullanıldığı reaksiyon türlerinden biridir. Aslında, SuzukiMiyaura çapraz-eşleşme reaksiyonu, en çok çalışılan çapraz-eşleşme reaksiyonlarından biridir. $\mathrm{Bu}$ reaksiyonda, bağlanması güç olan aril-aril birleşmesi gerçekleşir. Bu önemli reaksiyon, tarımsal kimya, malzeme bilimi ve farmasötik kimyası için önemli bir bileşikler sınıfı olmasının yanı sıra çeşitli biarillerin sentezi için çok faydalı bir yöntemdir $[17,18]$. Genel olarak, Suzuki-Miyaura çapraz-eşleşme reaksiyonu, yeni geliștirilen Pd-NHC komplekslerinin aktif katalizörler olduğunu kanıtlamak için kullanılır [16]. Ayrıca, bu reaksiyon yeni ve zor substratları başarılı bir şekilde birleștirmek için de kullanılır.

Bu konuda yayınlanan son çalışmalarda, su gibi çevre dostu bir çözücü içerisinde Pd-PEPPSI komplekslerinin katalitik aktiviteleri incelendi. Bu komplekslerin sulu ortamda Suzuki-Miyaura çapraz-eşleşme reaksiyonunda oldukça yüksek aktivite sergiledikleri tespit edildi [7, 19-21]. Bu çalışmada, 2-morfolinoetil sübstitüye Pd-PEPPSI kompleksleri sentezlendi. Sentezlenen bu komplekslerin yapısı, ${ }^{1} \mathrm{H}$ NMR, ${ }^{13} \mathrm{C}$ NMR ve FTIR spektroskopisi ve elementel analiz teknikleri kullanılarak aydınlatıldı. Ayrıca, 2morfolinoetil sübstitüyentli Pd-PEPPSI komplekslerinin, arilboronik asit türevleri ile aril klorürlerin Suzuki-Miyaura çapraz-eşleşme reaksiyonundaki katalitik aktiviteleri incelendi. Sentezlenen bütün PdPEPPSI komplekslerinin, Suzuki-Miyaura çaprazeşleşme reaksiyonunda oldukça yüksek aktivite sergiledikleri gözlendi.

\section{Materyal ve Metot}

2-morfolinoetil sübstitüye edilmiş Pd-PEPPSI komplekslerinin 1a-d sentezlendiği NHC öncülleri [22-24], İnönü Üniversitesi Fen Edebiyat Fakültesi Kataliz Araştırma laboratuvarında sentezlendi. Bu komplekslerin 1a-d sentezleri, standart Schlenk tekniği kullanılarak, ısıtılarak kurutulmuş cam malzemede, inert bir atmosfer altında gerçekleştirildi. Diğer tüm reaktifler, ticari olarak Merck, Sigma-Aldrich, Alfa Aesar, Acros kimyasal satan şirketlerden temin edildi. Bu kimyasallar bir daha saflaştırılmadan kullanıldı. Sentezlenen PdPEPPSI komplekslerini 1a-d erime noktaları, bir Electrothermal-9200 erime noktası aparatı ile havaya açık cam kılcallarda ölçüldü. FTIR spektrumları, Perkin Elmer Spectrum 100 FTIR spektrometresi kullanılarak 400-4000 $\mathrm{cm}^{-1}$ aralığında kaydedildi. Proton $\left({ }^{1} \mathrm{H}\right)$, ve karbon $\left({ }^{13} \mathrm{C}\right)$ NMR spektrumları, bir iç referans olarak tetrametilsilan ile $\mathrm{CDCI}_{3}$ içinde 400 $\mathrm{MHz}\left({ }^{1} \mathrm{H}\right), 100 \mathrm{MHz}\left({ }^{13} \mathrm{C}\right)$ 'de çalışan bir Bruker AS 400 Merkur spektrometresi kullanılarak kaydedildi. Tüm katalitik reaksiyon sonuçları, GC-FID tarafından Agilent 6890 N GC sisteminde, 30 m uzunluğunda, $0,32 \mathrm{~mm}$ çapında ve 0,25 $\mu \mathrm{m}$ film kalınlığında bir HP5 kolonunda gözlemlendi. Kolon kromatografisi, silika jel 60 (70-230 por) kullanılarak yapıldı. Elementel analizler, İnönü Üniversitesi Bilimsel ve
Teknolojik Araştırma Merkezi (Malatya, TÜRKIYE) tarafından gerçekleştirildi.

\section{Bulgular}

\subsection{Sentez}

\subsubsection{Dibromo[1-metil-3-(2-morfolinoetil) benzimidazol-2-iliden]-3-kloropiridin palladyum(II), 1a'nın sentezi:}

Kompleks 1a; 1-metil-3-(2-morfolinoetil)benzimidazolyum bromür (130 mg, $0.4 \mathrm{mmol}$ ), paladyum klorür (71 mg, $0.4 \mathrm{mmol}$ ), potasyum bromür (95 mg, 0.8 mmol) ve potasyum karbonat (276 $\mathrm{mg}, 2 \mathrm{mmol}$ ) 3kloropiridin ( $4 \mathrm{~mL}$ ) içine eklendi. Reaksiyon karışımı, $80{ }^{\circ} \mathrm{C}$ 'de 16 saat karıștırıldı. 3-kloropiridin vakum altında buharlaştırılarak uzaklaştırıldı. Kalıntıya diklorometan eklendi. Diklorometan içinde çözünen kalıntı silika jel kolonundan (1 cm kalınlığında) geçirildi. Diklorometan, vakum altında buharlaştırıldı. Ürün sarı bir katı olarak elde edildi. Ham ürün, npentan ile yıkandı ve oda sıcaklığında diklorometan / n-pentan (1/2) karışımında yeniden kristalleştirildi $[25,26]$. Verim: \% 72 (180 mg). e.n.: 216-217 ${ }^{\circ} \mathrm{C}$; $v_{(\mathrm{CN})}: 1413 \mathrm{~cm}^{-1} \cdot \mathrm{C}_{19} \mathrm{H}_{23} \mathrm{Br}_{2} \mathrm{C} \mathrm{lN}_{4} \mathrm{OPd}$ için hesaplanan analiz sonucu: C: 36.51; H: 3.71; N: 8.96. Bulunan: C: 36.48; $\mathrm{H}: 3.73$; N: 8.98. ${ }^{1} \mathrm{H}$ NMR (400 $\mathrm{MHz}, \mathrm{CDCl}_{3}$ ) için $\delta$ ppm: $4.31\left(\mathrm{~s}, 3 \mathrm{H},-\mathrm{NCH}_{3}\right) ; 2.60\left(\mathrm{t}, 4 \mathrm{H}-\mathrm{NCH}_{2} \mathrm{CH}_{2} \mathrm{O}-, \mathrm{J}\right.$ : $4.0 \mathrm{~Hz}) ; 3.14\left(\mathrm{t}, 2 \mathrm{H},-\mathrm{NCH}_{2} \mathrm{CH}_{2} \mathrm{~N}_{\text {morfolin, }} \mathrm{J:} 8.0 \mathrm{~Hz}\right) ; 3.62$ $\left(\mathrm{t}, \quad 4 \mathrm{H}-\mathrm{NCH}_{2} \mathrm{CH}_{2} \mathrm{O}-, \quad J: 4.0 \mathrm{~Hz}\right) ; 4.89(\mathrm{t}, 2 \mathrm{H}-$ $\mathrm{NCH}_{2} \mathrm{CH}_{2} \mathrm{~N}_{\text {morfolin, }} \mathrm{J}$ : $8.0 \mathrm{~Hz}$ ); 7.18-7.75 (m, 6H, Ar- $\boldsymbol{H}$ ); 8.70 and 8.81 (d, s, $2 \mathrm{H} \mathrm{Ar-C} \boldsymbol{H}_{\text {piridin, }}$ J: $\left.4.0 \mathrm{~Hz}\right) .{ }^{13} \mathrm{C}$ NMR $\left(400 \mathrm{MHz}, \mathrm{CDCl}_{3}\right)$ için $\delta$ ppm: $34.3\left(-\mathrm{NCH}_{3}\right) ; 50.9(-$ $\left.\mathrm{NCH}_{2} \mathrm{CH}_{2} \mathrm{O}-\right) ; \quad 54.3 \quad\left(-\mathrm{NCH}_{2} \mathrm{CH}_{2} \mathrm{~N}_{\text {morfolin }}\right) ; 56.7$ $\left.\mathrm{NCH}_{2} \mathrm{CH}_{2} \mathrm{~N}_{\text {morfolin }}\right) ; 67.0\left(-\mathrm{NCH}_{2} \mathrm{CH}_{2} \mathrm{O}-\right) ; 111.0,111.2$, 123.3, 124.9, 133.0, 134.8, 135.2 ve 138.1 (Ar-C); 150.4 ve 151.2 (Ar- $\left.\boldsymbol{C}_{\text {piridin }}\right) ; 161.3$ (2- $\left.\boldsymbol{C}_{\text {karben }}\right)$.

\subsubsection{Dibromo[1-(2-morfolinoetil)-3-propil benzimidazol-2-iliden]-3-kloropiridin palladyum(II), 1b'nin sentezi:}

Kompleks 1b; 1a için kullanılan yöntemin aynısı kullanılarak hazırlandı. Fakat kompleks 1b, 1-metil3-(2-morfolinoetil)benzimida-zolium bromür yerine, 1-(2-morfolinoetil)-3-propil benzimidazolyum bromür (142 mg, $0.4 \mathrm{mmol}$ ) kullanılarak hazırlandı. Verim: \% 68 (178 mg). e.n.: 248-249 ${ }^{\circ} \mathrm{C}$; $v_{(\mathrm{CN})}$ : 1412 $\mathrm{cm}^{-1} . \quad \mathrm{C}_{21} \mathrm{H}_{27} \mathrm{Br}_{2} \mathrm{C} \quad \mathrm{IN}_{4} \mathrm{OPd}$ için hesaplanan analiz sonucu: C: 38.62; H: 4.17; N: 8.58. Bulunan: C: 38.59; $\mathrm{H}: 4.19$; $\mathrm{N}: 8.60 .{ }^{1} \mathrm{H}$ NMR (400 $\mathrm{MHz}, \mathrm{CDCl}_{3}$ ) için $\delta \mathrm{ppm}$ : $1.07\left(\mathrm{t}, 3 \mathrm{H}, \mathrm{CH}_{3} \mathrm{CH}_{2} \mathrm{CH}_{2-}, \mathrm{J}: 8.0 \mathrm{~Hz}\right) ; 2.19(\mathrm{q}, 2 \mathrm{H}$, $\mathrm{CH}_{3} \mathrm{CH}_{2} \mathrm{CH}_{2-}$, J: $\left.6.8 \mathrm{~Hz}\right) ; 2.64\left(\mathrm{~s}, 4 \mathrm{H}-\mathrm{NCH}_{2} \mathrm{CH}_{2} \mathrm{O}-\right) ; 3.17$ (s, 2H, - $\mathrm{NCH}_{2} \mathrm{CH}_{2} \mathrm{~N}_{\text {morfolin); }} 3.66$ (t, $4 \mathrm{H}-\mathrm{NCH}_{2} \mathrm{CH}_{2} \mathrm{O}-$ );

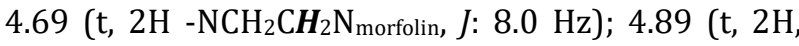
$\mathrm{NCH}_{2} \mathrm{CH}_{2} \mathrm{~N}_{\text {morfolin, }}$ J: $6.0 \mathrm{~Hz}$ ); 7.19-7.74 (m, 6H, Ar-H);

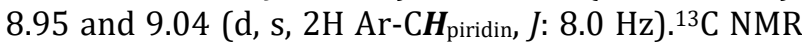
(400 MHz, $\left.\mathrm{CDCl}_{3}\right)$ için $\delta$ ppm: $11.8\left(\mathrm{CH}_{3} \mathrm{CH}_{2}-\right) ; 22.6$ $\left(\mathrm{CH}_{3}-\mathrm{CH}_{2}-\mathrm{CH}_{2}-\right) ; 46.1\left(\mathrm{~N}-\mathrm{CH}_{2} \mathrm{CH}_{2} \mathrm{CH}_{3}\right) ; 50.6\left(-\mathrm{N}-\mathrm{CH}_{2}-\right.$ $\left.\mathrm{CH}_{2}-\mathrm{O}-\right) ; \quad 54.0 \quad\left(-\mathrm{N}-\mathrm{CH}_{2} \mathrm{CH}_{2} \mathrm{~N}_{\text {morfolin }}\right) ; \quad 56.9 \quad$ ($\mathrm{NCH}_{2} \mathrm{CH}_{2} \mathrm{~N}_{\text {morfolin }}$ ); 66.9 (-N-CH2- $\mathrm{CH}_{2}-\mathrm{O}-$ ); 110.6, 110.7, 
123.1, 125.0, 132.7, 134.6, 135.0 ve 138.0 (Ar-C); 150.6 ve 151.7 (Ar- $\left.\boldsymbol{C}_{\text {piridin }}\right) ; 160.6$ (2- $\left.\boldsymbol{C}_{\text {karben }}\right)$.

\subsubsection{Dibromo[1-(2-morfolinoetil)-3-izopropil benzimidazol-2-iliden]-3-kloropiridin palladyum(II), 1c'nin sentezi:}

Kompleks 1c; 1a için kullanılan yöntemin aynısı kullanılarak hazırlandı. Fakat kompleks 1c, 1-metil-3(2-morfolinoetil)benzimidazolium bromür yerine, 1(2-morfolinoetil)-3-izopropil benzimidazolyum bromür (142 mg, $0.4 \mathrm{mmol}$ ) kullanılarak hazırlandı. Verim: \% 61 (159 mg). e.n.: 264-264 ${ }^{\circ} \mathrm{C}$; $v_{(\mathrm{CN})}$ : 1415 $\mathrm{cm}^{-1} . \quad \mathrm{C}_{21} \mathrm{H}_{27} \mathrm{Br}_{2} \mathrm{C} \quad \mathrm{IN}_{4} \mathrm{OPd}$ için hesaplanan analiz sonucu: C: 38.62; H: 4.17; N: 8.58. Bulunan: C: 38.64; $\mathrm{H}: 4.15$; N: 8.57. ${ }^{1} \mathrm{H}$ NMR (400 MHz, $\left.\mathrm{CDCl}_{3}\right)$ için $\delta \mathrm{ppm:}$ $1.73\left(\mathrm{~d}, 6 \mathrm{H}\left(\mathrm{CH}_{3}\right)_{2} \mathrm{CH}-, \mathrm{J}: 8.0 \mathrm{~Hz}\right) ; 2.62(\mathrm{t}, 4 \mathrm{H}(-$ $\mathrm{NCH}_{2} \mathrm{CH}_{2} \mathrm{O}-$ ) J: $4.0 \mathrm{~Hz}$ ); $3.12\left(\mathrm{t}, 2 \mathrm{H}-\mathrm{NCH}_{2} \mathrm{CH}_{2} \mathrm{~N}_{\text {morfolin, }} \mathrm{J}\right.$ : $8.0 \mathrm{~Hz}) ; 3.66$ (t, $4 \mathrm{H}-\mathrm{NCH}_{2} \mathrm{CH}_{2} \mathrm{O}-$, J: $\left.4.0 \mathrm{~Hz}\right) ; 4.86(\mathrm{t}, 2 \mathrm{H}$ $-\mathrm{NCH}_{2} \mathrm{CH}_{2} \mathrm{~N}_{\text {morfolin, }}$ J: 8.0Hz); $6.22\left(\mathrm{~m}, 1 \mathrm{H}-\mathrm{NCH}\left(\mathrm{CH}_{3}\right)_{2}\right)$; 7.16-7.74 (m, 6H Ar-H), 8.97 and 9.07 (s, 2H ArCH $\left.\boldsymbol{H}_{\text {piridin }}\right) .{ }^{13} \mathrm{C}$ NMR (400 $\left.\mathrm{MHz}, \mathrm{CDCl}_{3}\right)$ için $\delta \mathrm{ppm:} 20.7$ $\left(\left(\mathrm{CH}_{3}\right)_{2} \mathrm{CH}-\right) ; \quad 46.0 \quad\left(-\mathrm{NCH}_{2} \mathrm{CH}_{2} \mathrm{~N}_{\text {morfolin }}\right) ; \quad 54.1 \quad(-$ $\left.\mathrm{NCH}_{2} \mathrm{CH}_{2} \mathrm{O}-\right) ; \quad 55.0 \quad\left(-\mathrm{NCH}\left(\mathrm{CH}_{3}\right)_{2}\right) ; \quad 56.7 \quad(-$ $\left.\mathrm{NCH}_{2} \mathrm{CH}_{2} \mathrm{~N}_{\text {morfolin }}\right) ; 67.0\left(-\mathrm{NCH}_{2} \mathrm{CH}_{2} \mathrm{O}-\right) ; 111.0,112.4$, $122.7,122.8,125.0,132.4,132.7,136.0$ ve 138.1 (ArC); 150.6 ve 151.7 (Ar- $\left.\boldsymbol{C}_{\text {piridin }}\right) ; 159.7$ (2- $\left.\boldsymbol{C}_{\text {karben }}\right)$.

3.1.4. Dibromo[l-(2-morfolinoetil)-3-(2,3,5,6tetrametilbenzil)benzimidazol-2-iliden]-3-kloro piridinpalladyum(II), 1d'nin sentezi:

Kompleks 1d; 1a için kullanılan prosedürün aynısı kullanılarak hazırlandı. Fakat kompleks 1d, 1-metil3-(2-morfolinoetil)benzimidazolium bromür yerine, 1-(2-morfolinoetil)-3-(2,3,5,6-tetrametil benzil)benzimidazolyum bromür (184 $\mathrm{mg}, 0.4 \mathrm{mmol})$ kullanılarak hazırlandı. Verim: \% 59 (159 mg). e.n.: 227-229 ${ }^{\circ} \mathrm{C}$; $v_{(\mathrm{CN})}: 1411 \mathrm{~cm}^{-1} . \mathrm{C}_{29} \mathrm{H}_{35} \mathrm{Br}_{2} \mathrm{C} \mathrm{lN}_{4} \mathrm{OPd}$ için hesaplanan analiz sonucu: $\mathrm{C}: 45.99 ; \mathrm{H}: 4.66$; $\mathrm{N}$ 7.40. Bulunan: C: 46.01; H: 4.64; N: 7.43. ${ }^{1} \mathrm{H}$ NMR (400 MHz, $\left.\mathrm{CDCl}_{3}\right)$ için $\delta$ ppm: $2.15-2.17-2.19$ ve $2.20(\mathrm{~s}, 12 \mathrm{H}-$ $\left.\mathrm{C}_{6} \mathrm{H}\left(\mathrm{CH}_{3}\right)_{4}\right) ; 2.62\left(\mathrm{t}, 4 \mathrm{H}\left(-\mathrm{NCH}_{2} \mathrm{CH}_{2} \mathrm{O}-\right)\right.$ J: $\left.4.0 \mathrm{~Hz}\right) ; 3.16$

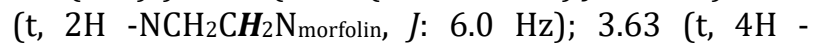
$\mathrm{NCH}_{2} \mathrm{CH}_{2} \mathrm{O}-$, J: $\left.4.0 \mathrm{~Hz}\right) ; 4.95\left(\mathrm{t}, 2 \mathrm{H}-\mathrm{NCH}_{2} \mathrm{CH}_{2} \mathrm{~N}_{\text {morfolin, }} \mathrm{J}\right.$ : $8.0 \mathrm{~Hz}) ; 6.17\left(\mathrm{~s}, 1 \mathrm{H}-\mathrm{C}_{6} \boldsymbol{H}\left(\mathrm{CH}_{3}\right)_{4}\right) ; 6.42-7.74(\mathrm{~m}, 6 \mathrm{H} \mathrm{Ar}-$ H), 8.82 ve 8.88 (s, d $2 \mathrm{H}$ Ar-CH piridin J: $8.0 \mathrm{~Hz}$ ). ${ }^{13} \mathrm{C}$ NMR (400 $\left.\mathrm{MHz}, \mathrm{CDCl}_{3}\right)$ için $\delta \mathrm{ppm}: 15.5$ ve 19.6 ($\left.\mathrm{C}_{6} \mathrm{H}\left(\mathrm{CH}_{3}\right)_{4}\right) ; \quad 49.5 \quad\left(-\mathrm{NCH}_{2} \mathrm{CH}_{2} \mathrm{~N}_{\text {morfolin }}\right) ; \quad 52.4$

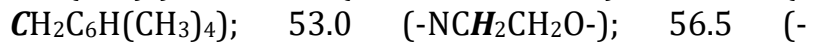
$\mathrm{NCH}_{2} \mathrm{CH}_{2} \mathrm{~N}_{\text {morfolin }}$; 66.0 (- $\left.\mathrm{NCH}_{2} \mathrm{CH}_{2} \mathrm{O}-\right)$; 109.4, 110.4, 121.9, 122.2, 123.8, 126.9, 127.1, 127.9, 129.5, 131.5, 131.6, $133.3,133.8,134.1$ and $137.1(\mathrm{Ar}-\boldsymbol{C}) ; 148.1$ ve 149.2 (Ar- $\left.\boldsymbol{C}_{\text {piridin }}\right) ; 162.1$ (2- $\left.\boldsymbol{C}_{\text {karben }}\right)$.

\subsection{Suzuki-Miyaura Çapraz-Eşleşme Reaksiyonu için Genel Yöntem:}

Literatürde tarif edildiği gibi, aril klorür (1 mmol), aril boronik asit (1.5 mmol), Pd-PEPPSI kompleksi 1a-d (0.01 mmol) ve $\mathrm{Cs}_{2} \mathrm{CO}_{3}(2 \mathrm{mmol}), \mathrm{H}_{2} \mathrm{O} / \mathrm{EtOH}$ (2/1) (3 mL) içinde havaya açık şekilde Schlenk tüpüne eklendi [20]. Tepkime karışımı $80^{\circ} \mathrm{C}$ 'de 2 saat karıștırıldı. Daha sonra oda sıcaklığına kadar soğutuldu. Organik faz, etilasetat / n-pentan (1/5) karışımı ile eksaktre edildi. Ekstrakte edilen karıșıma $\mathrm{MgSO}_{4}$ eklenerek, karışım kurutulup süzüldü. Elde edilen süzüntü, saf ürünü elde etmek üzere etilasetat / n-pentan (1/5) karıșımı kullanılarak 1 cm'lik silika jel kolon (60-120 por) ile saflaştırıldı. Çözücü vakum altında uzaklaștırıldı. Bileşiklerin saflığı gaz kromatografisi (GC) ile kontrol edildi. Dönüşümler, aril klorürlerin ürünlere dönüşümü dikkate alınarak hesaplandı.

\section{Tartışma ve Sonuç}

\subsection{Pd-PEPPSI komplekslerinin sentezi, 1a-d}

2-morfolinoetil sübstitüye edilmiş Pd-PEPPSI kompleksleri 1a-d; 2-morfolinoetil içeren NHC öncülleri (0.8 mmol), paladyum klorür $(0.8 \mathrm{mmol})$, potasyum bromür (1.6 mmol), potasyum karbonat ( 4 mmol) ve 3-kloropiridin (4 $\mathrm{mL})$ kullanılarak literatürde kullanılan yöntem ile sentezlendi $[25,26]$. Reaksiyon karıșımı, $80{ }^{\circ} \mathrm{C}$ 'de 16 saat karıștırıldı. PdPEPPSI kompleksleri 1a-d \%58 ile \%68 arasında verimle, açık sarı renkte bir katı halinde elde edildi. $\mathrm{Bu}$ çalışmada tanımlanan sentez Şekil 1'de gösterilmiștir. Hava ve neme kararlı Pd-PEPPSI kompleksleri 1a-d, dimetilformamid ve dimetilsülfoksit gibi polar çözücüler içinde iyi çözünürken, kloroform ve diklorometan gibi halojenli çözücüler içinde daha az çözündü. 2-morfolinoetil sübstitüyeli Pd-PEPPSI komplekslerinin 1a-d yapıları ${ }^{1} \mathrm{H}$ NMR, ${ }^{13} \mathrm{C}$ NMR ve FT-IR spektroskopik yöntemler ve elementel analiz teknikleri kullanılarak aydınla-tıldı. Bu spektrumlar önerilen formül ile tutarlıdır. Pd-PEPPSI komplekslerinin 1a-d sentezlendikleri NHC öncüllerinin ${ }^{1} \mathrm{H}$ NMR spektrumlarında düșük alanda 10.00 ve 11.00 ppm arasında gözlenen tuz pikleri, PdPEPPSI komplekslerinin $19-d \quad{ }^{1} \mathrm{H} \quad$ NMR spektrumlarında gözlenmedi. Ayrıca, Pd-PEPPSI komplekslerinin 1a-d ${ }^{1} \mathrm{H}$ NMR spektrumunda, 3kloropiridine ait 8.00 ve 9.00 ppm arasında gözlenen karakteristik dublet piki, bu bölgede gözlemlendi. PdPEPPSI komplekslerinin 1a-d ${ }^{13} \mathrm{C}$ NMR spektrumlarındaki Pd- $C_{\text {karben }}$ rezonanslarının, sırasıyla $\delta 161.3$, 160.6, 159.7 ve 162.1 ppm'e kaydı̆̆ı gözlendi. PdPEPPSI komplekslerinin $\mathbf{1 a - d}{ }^{13} \mathrm{C}$ NMR spektrumlarındaki $C_{\text {piridin }}$ rezonans pikleri sirasıyla 150.4151.2, 150.6-151.7, 150.6-151.7 ve 148.1-149.2 ppm'e kaydığı gözlendi. Pd-PEPPSI komplekslerinin 1a-d FT-IR verileri sirasiyla $v_{(\mathrm{CN})}$ : 1413, 1412, 1415 ve $1411 \mathrm{~cm}^{-1}$ de gözlendi. Bu verilerin tümü literatür ile uyumludur [19-21]. Elementel analiz sonuçları değerlendirildi. Hesaplanan değerlerin bulunan değerlere çok yakın olduğu gözlendi.

\section{2. Çeşitli arilboronik asit türevleri ile suzuki- miyaura çapraz-eşleşme reaksiyonu}

Sentezlenmiş Pd-PEPPSI komplekslerinin 1a-d katalitik aktiviteleri, Suzuki-Miyaura çapraz-eşleşme 
reaksiyonunda test edildi. Bu reaksiyon, biarillerin sentezinde kullanılan en önemli tepkimelerinden biridir. Ayrıca, bu tepkime diğer çapraz-eşleșme tepkimelerinden (Sonogashira, Stille, Kumuda, Negishi, Mizoroki-Heck, Himaya bağlanması gibi) daha yaygın kullanılır. $\mathrm{Bu}$ katalitik çalışmada, özellikle güvenli, çevre dostu, toksik olmayan ve ekonomik özelliğinden dolayı çözücü olarak su ve etil alkol karışımı kullanıldı [27, 28].

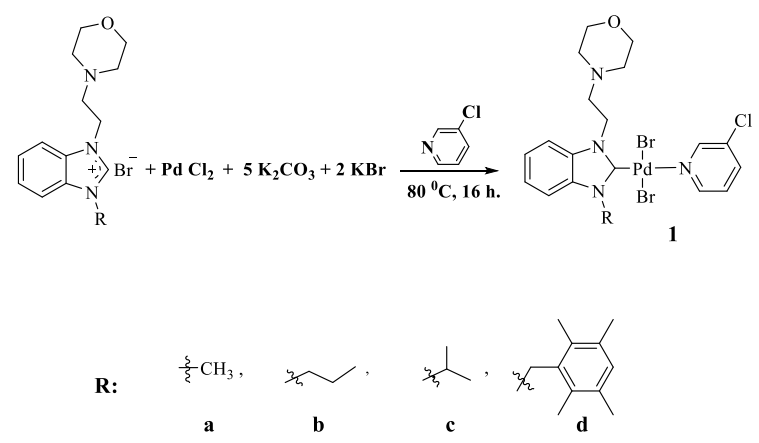

Şekil 1. 2-morfolinoetil sübsitüyeli Pd-PEPPSI komplekslerinin 1a-d sentezi

Genel olarak Pd-PEPPSI kompleksi 1d (hacimli sübstitüyentler içeren) yüksek katalitik aktivite gösterirken Pd-PEPPSI kompleksleri 1a-c (basit sübstitüyentler içeren) daha az katalitik aktivite gösterdiler. (Tablo 1-2). Kompleksler üzerindeki sübstitüyentlerin elektronik özelliklerinin ve sterik hacimlerinin katalitik aktivite üzerinde kısmen etkili olduğu gözlendi. Genelllikle, Pd-PEPPSI komplekslerinin 1a-d çok az yüklenmesi (\%1 mmol) ile hem elektronca zengin hem de elektronca fakir aril klorürlerin, her iki aril boronik asit türevi (fenilboronik asit ve 4-tertbütilfenilboronik asit) ile çapraz-eşleşmesi sonucu arzu edilen ürünler oldukça yüksek dönüşümde elde edildi (Tablo 1-2).

Bu çalışmada, substrat olarak 4-kloroasetofenon (asetat gibi elektron çeken grup içeren) ile 4kloroanisol (-metoksi gibi elektron verici grup içeren) karşılaştırıldı. 4-kloroasetofenonun kullanıldığı katalitik deneylerdeki dönüşümlerin, 4-kloroanisolun kullanıldığı katalitik deneylerdeki dönüşümlerden daha yüksek olduğu görüldü (Tablo 1-2). Benzer bir etki, fenilboronik asit ile 4-tertbütil fenilboronik asit arasında da görüldü. 4-tertbutil fenil boronik asidin (tert-butil gibi elektron verici grup içeren) kullanıldığı katalitik deneylerde dönüşümünlerin kısmen daha az olduğu gözlendi (Tablo 1-2).

Reaksiyon şartlarl; 4-arilklorür (1 mmol), fenilboronik asit (1.5 mmol), Pd-PEPPSI kompleksi 1a-d (0.01mmol) ve $\mathrm{Cs}_{2} \mathrm{CO}_{3}(2 \mathrm{mmol}), \mathrm{H}_{2} \mathrm{O} / \mathrm{EtOH}(2$ : 1) (3 mL) havaya açık şekilde Schlenk tüpüne eklendi. $80{ }^{\circ} \mathrm{C}$ 'de 2 saat karıștırıldı.

Bu çalışmada, çevre dostu çözücü sistemi olarak su ve etil alkol karışımı kullanıldı. Suzuki-Miyaura çaprazeşleşme reaksiyonu için su / etilalkol (2/1) çözücü sistemi içinde, aril klorürler (4-kloroasetofenon / 4- kloroanisol) ve boronik asit türevleri (fenilboronik asit / 4-tertbutil fenil boronik asit) kullanıldı. Fenil boronik asidin kullanıldığı katalitik deneylerde, ürün dönüşümleri \% 81 ile \% 98 arasında, 4-tertbutil fenil boronik asidin kullanıldığı katalitik deneylerde ise ürün dönüşümleri \% 74 ile \% 95 arasında gözlendi (Tablo 1-2).

Tablo 1. 2-morfolinoetil sübstitüyeli Pd-PEPPSI komplekslerinin 1a-d katalizörlügünde fenil boronik asit ve aril klorürlerin Suzuki-Miyaura çaparaz-eşleşme reaksiyonlarındaki dönüşümleri.

\begin{tabular}{|c|c|c|c|c|}
\hline Deney & R- & Ürün & Kat. & $\begin{array}{c}\text { Dönüşüm } \\
(\%)\end{array}$ \\
\hline 1 & & & $1 a$ & 91 \\
\hline 2 & $-\mathrm{COCH}$ & & $1 b$ & 89 \\
\hline 3 & Lan13 & 2 & $1 c$ & 95 \\
\hline 4 & & & 1d & 98 \\
\hline 5 & & & $1 \mathrm{a}$ & 84 \\
\hline 6 & $-\mathrm{OCH}_{3}$ & $\mathrm{H}_{3} \mathrm{CO}-$ & $1 b$ & 81 \\
\hline 7 & & 3 & 1c & 89 \\
\hline 8 & & & 1d & 92 \\
\hline
\end{tabular}

Tablo 2. 2-morfolinoetil sübstitüyeli Pd-PEPPSI komplekslerinin 1a-d katalizörlüğünde, 4-tertbutil fenil boronik asit ve aril klorürlerin Suzuki-Miyaura çaparazeşleșme reaksiyonlarındaki dönüşümleri.

\begin{tabular}{|c|c|c|c|c|}
\hline Deney & R- & Ürün & Kat. & $\begin{array}{c}\text { Dönüşüm } \\
(\%)\end{array}$ \\
\hline 1 & & & $1 a$ & 88 \\
\hline 2 & & & $1 b$ & 91 \\
\hline 3 & $\mathrm{CH}_{3}$ & 4 & 1c & 90 \\
\hline 4 & & & $1 d$ & 95 \\
\hline 5 & & & $1 a$ & 74 \\
\hline 6 & & & $1 b$ & 80 \\
\hline 7 & $-\mathrm{COCH}_{3}$ & 5 & $1 c$ & 78 \\
\hline 8 & & & 1d & 90 \\
\hline
\end{tabular}

İlk olarak, Pd-PEPPSI komp-lekslerinin 1a-d katalizörlügünde, fenil boronik asidin 4-kloroasetofenon ve 4-kloroanisol ile çapraz-eşleşme reaksiyonları incelendi. Arzu edilen $\mathbf{2}$ ve $\mathbf{3}$ nolu ürünler için dönüşümler sırasıyla \% 89-98 ve \% 81-92 arasında elde edildi (Tablo 1). Pd-PEPPSI kompleksi 1d'nin katalizörlüğünde 4-kloroasetofenon ile fenil boronik asidin çapraz-eşleşme ürünü 2, diğer Pd-PEPPSI komplekslerinin (1a-c) katalizör olarak kullanılmasından daha iyi bir dönüşümle (\% 98) elde edildi (Tablo 1). Pd-PEPPSI kompleksi 1d'nin katalizörlüğünde 4-kloroanisol ile fenil boronik asidin çapraz-eşleşme ürünü $\mathbf{3}$, diğer Pd-PEPPSI komplekslerinin (1a-c) katalizör olarak kullanılmasından daha iyi bir dönüşümle (\% 92) elde edildi (Tablo 1). Suzuki-Miyaura çapraz-eşleşme reaksiyonunda, 4-kloroasetofenon ile fenil boronik asit kullanıldığında, eşleşme ürünü 2'nin dönüşümlerinin eşleşme ürünü $\mathbf{3}$ 'ün dönüşümlerinden daha iyi olduğu gözlendi (Tablo 1). 4-kloroanisol ile fenil 
boronik asidin çapraz-eşleşme reaksiyonundan daha az dönüşümde ürün 3 elde edildi (Tablo 1).

İkinci olarak, 4-tertbutil fenil boronik asidin, 4kloroasetofenon ve 4-kloroanisol ile Pd-PEPPSI komplekslerinin 1a-d katalizörlüğünde, SuzukiMiyaura çapraz-eșleșme reaksiyonu incelendi. Arzu edilen 4 ve 5 ürünlerinin oluşumu incelendiğinde, dönüşümler sırasıyla \% 88-95 ve \% 74-90 arasında gözlendi (Tablo 2). Pd-PEPPSI kompleksi 1d'nin katalizörlüğünde 4-kloroasetofenon ile 4-tertbutil fenil boronik asidin çapraz-eșleșme ürünü 4'ün olușumu, diğer Pd-PEPPSI komplekslerinin (1a-c) katalizör olarak kullanılmasından daha iyi bir dönüşümle $(\%$ 95) elde edildi (Tablo 2). Pd-PEPPSI kompleksi 1d'nin katalizörlüğünde 4-kloroanisol ile 4-tertbutil fenil boronik asidin çapraz-eşleşme ürünü $\mathbf{5}^{\prime}$ in oluşumu, diğer Pd-PEPPSI komplekslerinin (1a-c) katalizör olarak kullanılmasından daha iyi bir dönüșümle (\% 90) elde edildi (Tablo 2). SuzukiMiyaura çapraz-eşleşme reaksiyonunda 4-tertbutil fenil boronik asit ile aril klorürler kullanıldığında, ürün 4'ün dönüşümünün, ürün $\mathbf{5}$ 'in dönüşümünden daha iyi olduğu gözlendi (Tablo 2). Bu çalışmada, 4kloroanisol ile 4-tertbutil fenil boronik asidin çaprazeșleşme ürünü $\mathbf{5}^{\prime}$ 'in, diğer tüm ürünlerin (2-4) dönüșümlerinden daha düșük olduğu gözlendi (Tablo 1-2).

Bu çalıșmada elde edilen sonuçlar, son zamanlarda çalışma grubumuz tarafından yayınlanan benzer bir çalışma ile karşılaştırıldığında katalizörlerin aktif oldukları gözlendi [19]. Başka bir çalışmada PEG zincirlerine bağlanan Pd(II)NHC komplekslerinin su / etilalkol çözücü karışımı içerisinde 24 saatteki katalitik aktivitesi ile karşılaştırıldığında yaklaşık katalitik dönüşümlerin elde edildiği görüldü [29]. Ayrıca, benzer bir çalışmada katalizör olarak manyetik nanopartikül destekli Pd(II)NHC kompleksleri, su / etilalkol çözücü ortamında daha kolay çapraz-eşleşme reaksiyonu veren aril bromürlerin biaril ürünlerine dönüşümleri 1 saat süre ile denenmiş ve yaklaşık sonuçlar elde edilmiștir [30]. Literatürde sentezlenen Pd-PEPPSI komplekslerinin Suzuki-Miyaura çapraz-eşleşmeleri, su / izopropil alkol çözücü karışımında daha kolay eşleşme reaksiyonu veren aril bromürlerle gerçekleștirilmiş ve bu çalışma ile benzer dönüşümler elde ediliştir [20, 31]. Bununla birlikte, Suzuki-Miyaura çapraz-eşleşme reaksiyonu için çözücü olarak sadece suyun kullanıldığı çalışma da bulunmaktadır [32]. Ancak çözücü olarak sadece suyun kullanıldığı çalıșmada, daha yüksek sıcaklıkta ve daha uzun sürede düșük verimler elde edilmiștir. $\mathrm{Bu}$ çalışmada, çevre dostu su / etil alkol çözücü karışımı kullanılarak, daha ılıman koşullarda (2 saat süre ve $80{ }^{\circ} \mathrm{C}$ sicaklık) aril klorürlerin biarillere yüksek dönüşümleri gerçekleştirildi. $\mathrm{Bu}$ nedenle, oldukça aktif katalizörler olan 2-morfolinoetil sübstitüyeli Pd-PEPPSI kompleksleri 1a-d sentezlendi. Sentezlenen kompekslerin su / etilalkol çözücü sisteminde, Suzuki-Miyaura çapraz-eşleşme reaksi- yonundaki katalitik aktiviteleri incelendi. Sentezlenen komplekslerin Suzuki-Miyaura çapraz-eşleşme reaksiyonunda aktif aktif katalizörler olduğu tespit edildi.

\section{Sonuç}

Sonuç olarak, Pd-PEPPSI kompleksleri 1a-d; 2morfolinoetil sübsitüyeli NHC öncüleri, paladyum klorür ve 3-kloropiridin'den sentezlendi. Bu komplekslerin yapılarl, ${ }^{1} \mathrm{H} \quad \mathrm{NMR},{ }^{13} \mathrm{C} \quad \mathrm{NMR}, \quad$ FTIR spektroskopisi ve elementel analiz teknikleri kullanılarak karakterize edildi. Suzuki-Miyaura çaprazeşleşme reaksiyonlarıda, 2-morfolinoetil sübstitüyeli Pd-PEPPSI komplekslerinin 1a-d katalitik aktiviteleri inceledi. Bütün komplekslerin Suzuki-Miyaura çapraz-eşleşme reaksiyonlarıda odukça aktif oldukları görüldü. Ayrıca katalitik çalışmalarda yeșil kimya açısından önemli olan çevre dostu çözücü sistemi su / etilalkol $\left(\mathrm{H}_{2} \mathrm{O} / \mathrm{EtOH} 2: 1\right)$ karışımı kullanıldı.

\section{Teşekkür}

Yazar, ${ }^{1} \mathrm{HNMR},{ }^{13} \mathrm{CNMR}$ ve FTIR spektroskopisi verilerinin teminine katkı sunan İnönü Üniversitesi Fen Edebiyat Fakültesi bünyesinde kurulan Kataliz Araştırma ve Uygulama Merkezi (İNÜ-KATAUM) Müdürlüğü'ne teşekkür eder. Elementel analizi ve GC (Gaz kromotografisi) analizi sonuçları için İnönü Üniversitesi Bilimsel ve Teknolojik Araștırma Merkezi'ne (İBTAM) teşekkür eder.

\section{Kaynakça}

[1] Bertogg, A., Campanaovo, F., Togni, A., 2005. NFerrocenyl-Substituted Planar-Chiral NHeterocyclic Carbenes and Their PdII Complexes. European Journal of Inorganic Chemistry, 2005(2), 347-356.

[2] Valente, C., Belowich, M.E., Hadei, N., Organ, M.G., 2010. Pd-PEPPSI Complexes and the Negishi Reaction. European Journal of Inorganic Chemistry, 2010(23), 4343-4354.

[3] Aktaş, A., Akkoç, S. Gök, Y. 2013. Palladium catalyzed Mizoroki-Heck and Suzuki-Miyaura reactions using naphthalenomethyl-substituted imidazolidin-2-ylidene ligands in aqueous media. Journal of Coordination Chemistry, 66(16), 2901-2909.

[4] Cinderella, A.P., Vulovic, B., Watson, D.A., 2017. Palladium-Catalyzed Cross-Coupling of Silyl Electrophiles with Alkylzinc Halides: A SilylNegishi Reaction. Journal of the American Chemical Society, 139(23), 7741-7744.

[5] Cordovilla, C., Bartolomé, C., Martínez-Ilarduya, J.M., Espinet P., 2015. The Stille Reaction, 38 Years Later. ACS Catalysis, 5(5), 3040-3053.

[6] Aktaş, A. Barut Celepci, D., Gök, Y., Aygün, M. 2018. 2-Hydroxyethyl-Substituted (NHC)Pd(II) PPh3 Complexes: Synthesis, Characterization, 
Crystal Structure and Its Application on Sonogashira Cross-Coupling Reactions in Aqueous Media. ChemistrySelect (3),1093210937

[7] Osi'nska, M., Gniewek, A., Trzeciak, A.M., 2016. Suzuki-Miyaura, Hiyama coupling catalyzed by PEPPSI-type complexes with non-bulky NHC ligand. Journal of Molecular Catalysis A: Chemical, (418-419) C.S., Bhattacharyya, A., Hwang, W.S., Lin, I.J.B., 2009. Coinage Metal-NHeterocyclic Carbene Complexes. Chemical Reviews, 109(8), 3561-3598.

[8] Sarı, Y., Aktaş, A., Barut Celepci, D., Gök, Y., Aygün, M., 2017. Synthesis, Characterization and Crystal Structure of New 2-MorpholinoethylSubstituted Bis-(NHC)Pd(II) Complexes and the Catalytic Activity in the Direct Arylation Reaction. Catalysis Letters 147(9), 2340-2351.

[9] Gök, Y., Aktas, A., Erdoğan, H., Sarı, Y., 2018. New 4-vinylbenzyl-substituted bis(NHC)-Pd(II) complexes: Synthesis, characterization and the catalytic activity in the direct arylation reaction. Inorganica Chimica Acta, (471), 735-740.

[10] Chen, W.-X., Zhang, C.-Y., Lu, J.-M., 2013. Highly efficient amination in neat water of benzyl chlorides with dialkylformamides catalysed by N-heterocyclic carbene-palladium(II)-1methylimi. Journal of Chemical Research, 37(10), 611-614.

[11] Poyatos, M., Mata, J.A., Peris, E., 2009. Complexes with Poly(N-heterocyclic carbene) Ligands: Structural Features and Catalytic Applications. Chemical Reviews, 109(8), 3677-3707.

[12] Díez-González, S., Marion, N., Nolan, S.P., 2009. $\mathrm{N}$-Heterocyclic Carbenes in Late Transition Metal Catalysis. Chemical Reviews, 109(8), 3612-3676.

[13] Zhu, L., Ye, Y., Shao L., 2012. Well-defined NHC$\mathrm{Pd}(\mathrm{II})-\mathrm{Im}(\mathrm{NHC}=\mathrm{N}-$ heterocyclic carbene; $\mathrm{Im}=1$ methylimidazole) complex catalyzed $\mathrm{C}-\mathrm{N}$ coupling of primary amines with aryl chlorides. Tetrahedron, 68(10), 2414-2420.

[14] Inomata, H., Toh, A., Mitsui, T., Fukuzawa, S., 2013. N-heterocyclic carbene copper(I) complex-catalyzed direct $\mathrm{C}-\mathrm{H}$ thiolation of benzothiazoles. Tetrahedron Letters, 54(35), 4729-4731.

[15] Fortman, G.C., Nolan, S.P., 2011. N-Heterocyclic carbene (NHC) ligands and palladium in homogeneous cross-coupling catalysis: a perfect union. Chemical Society Reviews, 40(10), 51515169.

[16] Zhou, Z.-Z., Liu, F.-S., Shen, D.-S., Tan, C., Luo, L.Y., 2011. Efficient palladium-catalyzed Suzuki cross-coupling reaction with $\beta$-ketoamine ligands. Inorganic Chemistry Communications, 14(5), 659-662.
[17] Polshettiwar, V., Decottignies, A., Len, C., Fihri, A., 2010. Suzuki-Miyaura Cross-Coupling Reactions in Aqueous Media: Green and Sustainable Syntheses of Biaryls. ChemSusChem 3(5), 502-522.

[18] Benhamou, L., Besnar., C., Kündig, E.P., 2014. Chiral PEPPSI Complexes: Synthesis, Characterization, and Application in Asymmetric Suzuki-Miyaura Coupling Reactions. Organometallics, 33(1), 260-266.

[19] Aktaş, A., Barut Celepci, D., Gök, Y., Aygün, M. 2018. 2-Hydroxyethyl-Substituted Pd-PEPPSI Complexes: Synthesis, Characterization and the Catalytic Activity in the Suzuki-Miyaura Reaction for Aryl Chlorides in Aqueous Media. ChemistrySelect (3), 9974-9980.

[20] Touj, N., Gürbüz, N., Hamdi, N., Yaşar, S., Özdemir, İ., 2018. Palladium PEPPSI complexes: Synthesis and catalytic activity on the SuzukiMiyaura coupling reactions for aryl bromides at room temperature in aqueous media. Inorganica Chimica Acta, (478), 187-194.

[21] He, X.-X., Li, Y., Ma, B.-B., Ke, Z., Liu, F.-S., 2016. Sterically Encumbered Tetraarylimidazolium Carbene Pd-PEPPSI Complexes: Highly Efficient Direct Arylation of Imidazoles with Aryl Bromides under Aerobic Conditions. Organometallics, 35(16), 2655-2663.

[22] Özdemir, İ., Gök, Y., Gürbüz, N., Çetinkaya, E., Çetinkaya, B., 2004. Palladium-catalyzed Suzuki reaction using 1,3-dialkylbenzimidazol-2ylidene ligands in aqueous media. Heteroat. Chem., 15(6), 419-423.

[23] Çekirdek, S., Yaşar, S., Özdemir, İ., 2014. Palladium(II)-N-heterocyclic carbene complexes: synthesis, characterization and catalytic application. Appl. Organomet. Chem., 28(6), 423-431.

[24] Aktaş, A., Keleştemur, Ü., Gök, Y., Balcıoğlu, S., Ateş, B., Aygün, M., 2018. Morpholinoethyl-substituted N-heterocyclic carbene (NHC) precursors and their silver(I)NHC complexes: synthesis, crystal structure and in vitro anticancer properties. J. Iran. Chem. Soc. 15, 131-139.

[25] Barbu, L., Popa, M.M., Shova, S., Ferbinteanu, M., Draghici, C., Dumitrascu, F., 2017. New Pd-NHC PEPPSI complexes from benzimidazolium salts with a phenacyl group attached to N3. Inorganica Chimica Acta, (463), 97-101.

[26] Seva, L., Hwang, W.S., Sabiah, S., 2016. Palladium biphenyl N-heterocyclic carbene complexes: Synthesis, structure and their catalytic efficiency in water mediated Suzuki-Miyaura crosscoupling reaction. Journal of Molecular Catalysis A: Chemical, (418-419), 125-131. 
[27] Turkmen, H., Pelit, L., Cetinkaya, B., 2011. Watersoluble cis-[(NHC)PdBr2(TPPTS)] catalysts and their applications in Suzuki-Miyaura coupling of aryl chlorides. Journal of Molecular Catalysis A: Chemical, 348(1-2), 88-93.

[28] Shaughnessy, K.H., 2009. Hydrophilic Ligands and Their Application in Aqueous-Phase MetalCatalyzed Reactions. Chemistry Revviews. 109(2), 643-710.

[29] Schroeter, F., Soellner, J., Strassner. T., 2018. Cyclometalated Palladium NHC Complexes Bearing PEG Chains for Suzuki-Miyaura CrossCoupling in Water. Organometallics 37(22), 4267-4275.

[30] Vishal, K., Fahlman, B.D., Sasidhar, B.S., Patil, S.A., Patil. S.A., 2017. Magnetic Nanoparticle-
Supported N-Heterocyclic CarbenePalladium(II): A Convenient, Efficient and Recyclable Catalyst for Suzuki-Miyaura CrossCoupling Reactions. Catal Lett 147(4), 900-918.

[31] Şahin, N., 2019. PEPPSI-type 2-methyl-2propenyl-functionalized N-heterocyclic carbenepalladium complexes: Synthesis, structural characterization and catalytic activity on SuzukieMiyaura reaction. Journal of Molecular Structure 1177, 193-198.

[32] Shi, J.-C., Yu, H., Jiang, D., Yu, M., Huang, Y., Nong, L., Zhang, Q., Jin. Z., 2014. N-Heterocyclic Carbene Conjugated with Poly(ethylene glycol) for Palladium-Catalyzed Suzuki-Miyaura Coupling in Aqueous Solvents. Catal Lett 144(1), 158-164. 